\title{
Loss of vision in one eye following scoliosis surgery
}

\author{
J West, G Askin, M Clarke, S A Vernon
}

\begin{abstract}
A 50-year-old woman developed left sided total external ophthalmoplegia and complete visual loss, with evidence of choroidal non-perfusion, immediately following scoliosis surgery. The ocular movements recovered, but the eye remained blind. It is suggested that these lesions may have resulted from pressure on the orbital contents during surgery.
\end{abstract}

The case is reported of a 50-year-old woman who developed unilateral total external ophthalmoplegia and blindness following scoliosis surgery.

\section{Case history}

A 50-year-old nurse was seen at the back pain clinic in June 1986. She had undergone a spinal fusion for scoliosis at the age of 15 but had suffered persistent pain and intermittent claudication since. In April 1987 she was operated on for decompression at levels $\mathrm{L} 1, \mathrm{~L} 2$, and L 3 followed by stabilisation with Harrington distraction rods, supplemented by wires passed under the lamina and secured to the rods.

Anaesthetic induction was with propofol, fentanyl, and droperidol. Atracurium (total dose $55 \mathrm{mg}$ ), fentanyl (total dose $350 \mathrm{mg}$ ), chlorpromazine (total dose $7.5 \mathrm{mg}$ ) and labetalol (total dose $10 \mathrm{mg}$ ) were given at intervals throughout the operation. During the operation she was positioned prone with the eyes covered by a gauze swab and tape. The anaesthetic time was $4 \frac{1}{2}$ hours. Care was taken to avoid pressure on the eyes. Total blood loss was estimated at 1 litre, and the systolic blood pressure was maintained at between 70 and $90 \mathrm{mmHg}$ throughout the procedure.

Immediately after the operation the patient

Department of

Ophthalmology, Queen's

Medical Centre,

J West

M Clarke

S A Vernon

Department of Orthopaedic Surgery G Askin

Correspondence to: M Clarke, FRCS

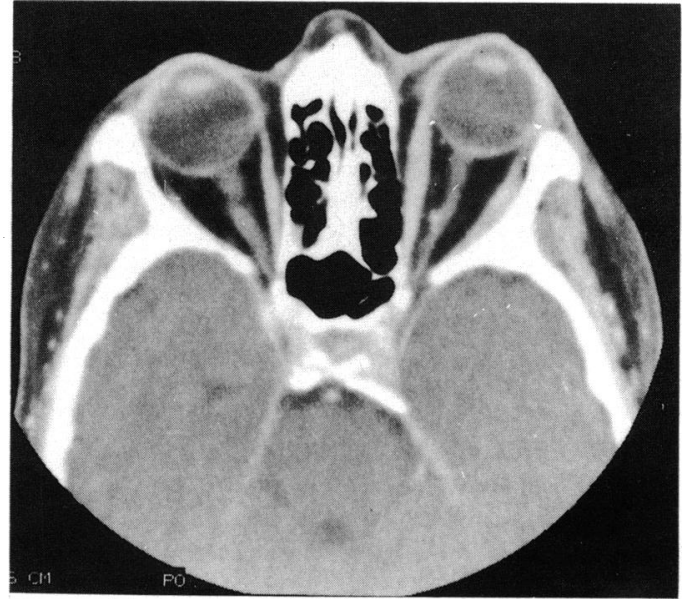

Figure 1: C T scan one week after operation showing left proptosis and swelling of left medial rectus but no abnormality in the cavernous sinus. noted complete, painless visual loss in the left eye. The left orbit was observed to be congested and the left eye proptosed. External ocular movements were absent. The left corneal reflex was reduced. The left pupil was fixed and middilated. The left optic disc was pale and the retinal vessels attenuated. The right eye was normal.

Cavernous sinus thrombosis was suspected, but not confirmed either by $\mathrm{C} \mathrm{T}$ or magnetic resonance imaging (Fig 1). Treatment was instituted with intravenous antibiotics and anticoagulants. Three months later the left eye had no perception of light and was divergent and hypertropic. External ocular movements had recovered, but adduction was painful and limited. The left corneal reflex was reduced. There was no iris atrophy. Examination of the fundus showed evidence of choroidal and retinal non-perfusion (Fig 2).

\section{Discussion}

Sudden loss of vision following general and orthopaedic surgery has been reported, ${ }^{12}$ but to our knowledge no previous case has been associated with ophthalmoplegia or such striking involvement of the choroidal circulation. In previous cases the mechanism of visual loss was infarction of the retina or optic nerve following hypotension or embolism. The ophthalmoplegia seen in our patient could have been due to cavernous sinus thrombosis. However, this rarely causes permanant loss of vision, ${ }^{3}$ and there was no evidence of thrombosis on MRI scan.

Hollenhorst et al reported eight cases of unilateral blindness following neurosurgical procedures in $1954 .{ }^{4}$ These patients either lay prone or were sitting with the face cushioned on a headrest. The clinical features in these patients were similar to our own case. They reproduced the lesion experimentally in monkeys using a combination of hypotension and pressure on the orbit.

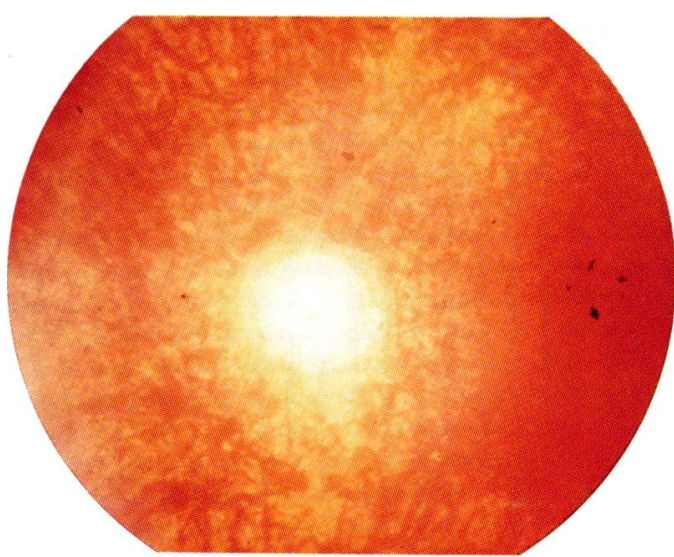

Figure 2: Left optic disc showing pallor and loss of retinal and choroidal vessels. 
Acute ischaemic lesions involving the choroid are rare. This rarity is thought to be because of communications between the adjacent choroidal segments via the vortex veins. ${ }^{5}$ Amalric reported the case of a 34-year-old woman who lost the vision of one eye following mammoplasty. ${ }^{6}$ The fundal appearances were similar to those in our case. There had been no perioperative hypotension, and perioperative ocular compression was thought to be the cause. We believe this to be the likely cause of injury in our patient. Prolonged compression of the orbital contents during anaesthesia can give rise to ocular and orbital ischaemia, causing blindness and ophthalmoplegia, and adequate protection should be given to the eyes during procedures where such compression might occur.

We thank Dr A M Whiteley and Mr J Webb for helpful discussion and permission to report this case and Dr T Jaspan for interpreting the radiographs.

1 Rizzo JF, Lessell S. Posterior ischaemic optic neuropathy during general surgery. Am f Ophthalmol 1987; 103: 808-11. 2 Sweeney PJ, Breuer AC, Selhorst JB et al. Ischaemic optic neuropathy: a complication of cardiopulmonary bypass surgery. Neurology 1982; 32: 560-2.

3 Walsh FB, Hoyt WT. Clinical neuroophthalmology. 3rd ed. Baltimore: Williams and Wilkins, 1984: 1892-4.

4 Hollenhorst RW, Svien HJ, Benoit CF. Unilateral blindness occurring during anaesthesia for neurosurgical operations. Arch Ophthalmol 1954; 52: 819-30.

5 Hayreh SS. Acute choroidal ischaemia. Trans Ophthalmol Soc UK 1980; 100: 400-7. 6 Amalric P. Acute choroidal ischaemia. Trans Ophthalmol Soc
UK 1971; 91: 305-22. 